\section{Ejercicio o prescripción dietética: efectos sobre la salud en mujeres inactivas con malnutrición por exceso}

\author{
FABIÁN RODRÍGUEZ-BRICEÑO ${ }^{1, \mathrm{a}}$, CLAUDIA MARTÍNEZ ESPINOZA ${ }^{1, \mathrm{~b}}$, \\ EDGARDO MOLINA-SOTOMAYOR ${ }^{2, c}$, \\ FERNANDO RODRÍGUEZ-RODRÍGUEZ ${ }^{3, c}$
}

\section{Effects of an exercise program or a dietary prescription in overweight or obese women}

Background: Physical activity and dietary prescription are the mainstay of overweight and obesity management. Aim: To compare the effects of physical activity or dietary management in overweight or obese sedentary women. Material and Methods: Thirty-four physically inactive adult women with overweight or obesity, were intentionally divided into three groups: controls $(C)$, exercise (E) and dietary prescription (DP). Women in the E group were trained in a circuit for twelve weeks and a hypocaloric diet was prescribed to those in the $D P$ group. Blood pressure, body composition and phase angle (FA) measured using bioimpedance and dynamometry, were measured at baseline and the end of the intervention. Results: Women in the E group had a significant increase in muscle mass and intracellular water and significant decreases in systolic and diastolic blood pressure levels. No significant differences in dynamometry were observed between groups. The DP group had significant decreases in body weight, fat mass, body mass index, waist circumference, systolic and diastolic blood pressure. Conclusions: The DP group had a higher weight reduction than the E group. Dietary prescription should be complemented with a physical activity program in the management of overweight or obesity.

(Rev Med Chile 2020; 148: 469-477)

Key words: Exercise; Diet, Healthy; Obesity.

\author{
'Escuela de Salud, Universidad \\ Tecnológica de Chile INACAP. \\ Valparaíso, Chile. \\ 2Departamento de Educación \\ Física. Universidad Metropolitana \\ de Ciencias de la Educación. \\ Santiago, Chile. \\ ${ }^{3}$ Grupo IRyS, Escuela de \\ Educación Física, Pontificia \\ Universidad Católica de \\ Valparaíso. Valparaíso, Chile. \\ aLicenciado en Nutrición, \\ Magíster en Actividad Física para \\ la Salud. \\ 'Licenciada en Enfermería. \\ 'Profesor de Educación Física, \\ Doctor en Ciencias de la Actividad \\ Física.
}

Trabajo financiado por la Escuela de Salud de la Universidad

Tecnológica de Chile INACAP Valparaíso.

Los autores declaran no tener conflictos de interés.

Recibido el 21 de octubre de 2019, aceptado el 6 de abril de 2020.

Correspondencia a: Fernando Rodríguez-Rodríguez Avda. El Bosque 1290, Santa Inés, Viña del Mar, Chile. fernando.rodriguez@pucv.cl
A ctualmente, la malnutrición por exceso, sobrepeso (SP) u obesidad (OB), está considerada como enfermedad crónica, siendo uno de los mayores contribuyentes a la carga de enfermedad, discapacidad y muerte prematura ${ }^{1}$.

La prevalencia de exceso de peso, según la última Encuesta Nacional de Salud (ENS) 20162017 en la población > 15 años, es de 74,2\%, donde $31,2 \%$ presenta OB, 39,8\% presenta SP y 3,2\% está en el rango de OB mórbida, cifras superiores a la ENS 2009-2010², que mostró 67\% de la población adulta con exceso de peso. Este aumento, se explicaría, en gran medida, por la conducta sedentaria, inactividad física y cambios en los estilos de alimentación.

En Chile, según la última Encuesta Nacional de Hábitos de Actividad Física y Deporte 2018, en población $>18$ años, $81,3 \%$ es inactiva físicamente, siendo significativamente mayor en mujeres $(74,2 \%)$ que en hombres $(54,7 \%)^{3}$.

Existen diferentes estrategias de intervención para enfrentar la malnutrición por exceso, siendo la actividad física y la prescripción dietética fundamentales para tratar esta patología.

La mayoría de las intervenciones basadas en alimentación y actividad física se enfocan, principalmente, en reportes de pérdida de peso 
corporal ${ }^{4-6}$, siendo menos estudiados los cambios en la composición corporal, por la gran variedad de técnicas para determinar la proporción entre tejidos, dificultando así la comparación entre investigaciones ${ }^{7}$.

Si bien la literatura sugiere una prescripción dietética combinada con un programa de actividad física para mejorar la composición corporal ${ }^{8}$, también es importante evaluar qué ocurre con los compartimentos corporales y otros indicadores de salud cuando estas estrategias de intervención se aplican en forma independiente.

El objetivo de este estudio fue comparar los efectos de un programa de actividad física o de prescripción dietética, sobre la composición corporal y otros indicadores de salud en mujeres adultas, físicamente inactivas, con malnutrición por exceso.

\section{Métodos}

\section{Participantes}

Este estudio de intervención cuasiexperimental y longitudinal incluyó a 34 mujeres adultas, administrativas en una universidad chilena (UTC INACAP, Valparaíso, Chile).

Las participantes tenían entre 25 y 59 años, con SP u OB, físicamente inactivas y aceptaron participar voluntariamente en el estudio. Previamente firmaron un consentimiento informado, que explicaba las características del estudio, riesgos, beneficios y que garantizaba la confidencialidad de los datos. El estudio fue aprobado por el Comité de Bioética de la Pontificia Universidad Católica de Valparaíso, con referencia BIOEPUCV-H154-2018.

Se excluyó a embarazadas y a aquellas con desfibriladores implantados o marcapasos. Además, fueron excluidas las mujeres con estado nutricional normal o bajo peso, físicamente activas, o bien, que estuviesen con indicación de alguna prescripción dietética que indujera cambios en el peso corporal durante el estudio.

\section{Variables del estudio}

Respecto a la composición corporal, se evaluó peso, estatura, índice de masa corporal (IMC), perímetro de cintura, masa grasa (MG), masa muscular (MM), agua intracelular (AIC), agua extracelular (AEC) y agua corporal total (ACT).
También, se consideraron como variables el ángulo de fase $(\mathrm{AnF})$, la dinamometría manual y presión arterial (PA).

La muestra se distribuyó de manera intencional en tres grupos: grupo control (GC), grupo experimental con actividad física (GE) y grupo experimental con prescripción dietética (GPD).

Para realizar las mediciones de peso y estatura, se siguió el protocolo establecido por la International Society for the Advancement of Kinanthropomety $(\text { ISAK })^{9}$. La evaluación de bioimpedancia (BIA) se ajustó a las normas estandarizadas ${ }^{10}$, donde la persona debía estar en decúbito supino, en ayuno $>4$ horas, no haber hecho ejercicio físico al menos 24 horas antes y no haber ingerido alcohol desde el día anterior. Respecto a la dinamometría manual, la persona, de pie y con el brazo extendido paralelamente al tronco, sujetaba el dinamómetro y ejercía la fuerza máxima de presión. Este procedimiento fue repetido dos veces, alternando mano derecha e izquierda, registrándose como medida válida el mejor de los dos intentos. Para la medición de PA, se siguió la técnica estandarizada de la Guía Clínica de Hipertensión Arterial primaria en personas $>15$ años $^{11}$.

\section{Instrumentos}

Para la composición corporal, se utilizó una báscula mecánica con tallímetro Seca modelo 700 (Seca, Hamburgo, Alemania). Las otras variables como agua corporal y AnF, se obtuvieron a partir de un analizador octopolar y multifrecuencia de BIA InBody S10 (InBody Co. Seúl, Corea) de 6 frecuencias de medición $(1 \mathrm{kHz}, 5 \mathrm{kHz}, 50 \mathrm{kHz}$, $250 \mathrm{kHz}, 500 \mathrm{kHz}, 1 \mathrm{MHz})$.

Para determinar la fuerza estática de presión manual, se utilizó un dinamómetro digital marca Baseline modelo 12-0286 (Baseline Evaluation Instruments, White Plains, NY, USA) y para la medición de PA un medidor digital marca Nissei modelo DSK-1011 (Nihon Seimitsu Sokki Co. Ltd., Gunma, Japón).

\section{Programa de actividad física}

El GE fue sometido a un entrenamiento en circuito realizado por un profesor de Educación Física. El programa tuvo un total de 36 sesiones de $50 \mathrm{~min}$, dividida en $10 \mathrm{~min}$ de calentamiento, 30 min de estaciones de trabajo (parte principal) y 10 min de vuelta a la calma, por 3 días/semana durante 12 semanas. 
Ejercicio o prescripción dietética sobre la salud en mujeres - F. Rodríguez-Briceño et al

El calentamiento consistió en ejercicios de movilidad articular y elongación muscular con intensidad entre 11-12 en escala de Borg. La parte central consistió en la realización de diferentes ejercicios, comenzando con 4 series de 20 seg de trabajo por 30 seg de descanso y 2 min de recuperación entre cada vuelta al circuito, aumentando el número de series, tiempo de trabajo y disminuyendo el descanso de manera progresiva cada 2 semanas. La parte central se realizó a una intensidad de 15-16 en escala de Borg. La vuelta a la calma consistió en ejercicios de relajación y flexibilidad de miembros inferiores y miembros superiores con intensidad moderada entre 11-12 en escala de Borg.

El programa tuvo 2 fases: de adaptación, desde la semana 1 a 5 , entre 40 y $50 \%$ de la frecuencia cardiaca de reserva $(\mathrm{FcR})$ y la segunda fase, de mejora de la condición física, desde la semana 6 a la 12 , entre 45 y $65 \%$ de la FcR (Tabla 1).

Se decidió usar el \% FcR, ya que ha sido aceptado como equivalente al \% $\mathrm{VO}_{2 \max }$ (ACSM, 2006). Para el cálculo de la FCmáx se utilizó una estrategia de cálculo indirecto, a través de la fórmula propuesta por Tanaka para mujeres: 208,1 - (0,77 $\mathrm{x}$ edad en años) $)^{35}$. Para el cálculo del porcentaje de la $\mathrm{FcR}(\% \mathrm{FcR})$, se utilizó la fórmula propuesta por Karvonen: Intensidad del ejercicio en $\% \mathrm{x}$ FcR + FCrep ${ }^{36}$.

\section{Prescripción dietética}

El GPD siguió un régimen hipocalórico, hiperproteico, hipograso y fraccionado, durante 12 semanas, de manera progresiva. La prescripción

Tabla 1. Características de las sesiones del grupo ejercicio

\begin{tabular}{|c|c|c|c|}
\hline Fase & Semana & Actividad & Intensidad \\
\hline \multirow[t]{5}{*}{ Fase 1} & 1 & $\begin{array}{l}\text { Circuitos } 6 \text { estaciones (puente en decúbito supino, sentadillas, flexiones de codo, tijeras } \\
\text { laterales, puente lateral y caminata estática) }\end{array}$ & \multirow[t]{5}{*}{$40-50 \%$ FcR } \\
\hline & 2 & $\begin{array}{l}\text { Circuitos } 6 \text { estaciones (puente en decúbito supino, flexiones de codo, tijeras laterales, } \\
\text { puente lateral, estocadas frontales y tríceps con banda elástica) }\end{array}$ & \\
\hline & 3 & $\begin{array}{l}\text { Circuitos } 6 \text { estaciones (desplazamientos laterales con banda elástica entre muslos, flexio- } \\
\text { nes de codo, saltos con piernas separadas, puente lateral, estocadas frontales y tríceps } \\
\text { con banda elástica) }\end{array}$ & \\
\hline & 4 & $\begin{array}{l}\text { Circuitos } 6 \text { estaciones (puente en decúbito supino, flexiones de codo, tijeras laterales, } \\
\text { balanceo con pesa rusa, doble puente y tríceps con banda elástica) }\end{array}$ & \\
\hline & 5 & $\begin{array}{l}\text { Circuitos } 6 \text { estaciones (puente en decúbito supino, flexiones de codo, tijeras laterales, } \\
\text { balanceo con pesa rusa, "superman" y remo sentado con banda elástica) }\end{array}$ & \\
\hline \multirow[t]{7}{*}{ Fase 2} & 6 & $\begin{array}{l}\text { Circuitos } 6 \text { estaciones (puente en decúbito supino, flexiones de codo, tijeras laterales, } \\
\text { balanceo con pesa rusa, jumping jacks y remo sentado con banda elástica) }\end{array}$ & \multirow[t]{5}{*}{$45-55 \%$ FcR } \\
\hline & 7 & $\begin{array}{l}\text { Circuitos } 8 \text { estaciones (puente en decúbito supino, flexiones de codo, tijeras laterales, bur- } \\
\text { pees, "superman", remo sentado con banda elástica, "patinador de hielo" y "yoga flow") }\end{array}$ & \\
\hline & 8 & $\begin{array}{l}\text { Circuitos } 8 \text { estaciones (puente doble, flexiones de codo, tijeras laterales, balanceo con pesa } \\
\text { rusa, "superman", remo sentado con banda elástica, "patinador de hielo" y "yoga flow") }\end{array}$ & \\
\hline & 9 & $\begin{array}{l}\text { Circuitos } 8 \text { estaciones ("Superman" con o sin apoyos, flexiones de codo, tijeras laterales, } \\
\text { burpees, estocadas peso corporal, remo sentado con banda elástica, "patinador de hielo" } \\
\text { y "yoga flow") }\end{array}$ & \\
\hline & 10 & $\begin{array}{l}\text { Circuitos } 8 \text { estaciones ("Superman" con o sin apoyos, flexiones de codo, tijeras laterales, } \\
\text { burpees, doble puente, remo sentado con banda elástica, "patinador de hielo" y "yoga } \\
\text { flow") }\end{array}$ & \\
\hline & 11 & $\begin{array}{l}\text { Circuitos } 8 \text { estaciones ("Superman" con o sin apoyos, flexiones de codo, tijeras laterales, } \\
\text { burpees, puentes laterales activos, remo sentado con banda elástica, "patinador de hielo" } \\
\text { y "goliat") }\end{array}$ & \multirow[t]{2}{*}{$45-65 \%$ FcR } \\
\hline & 12 & $\begin{array}{l}\text { Circuitos } 8 \text { estaciones ("Superman" con o sin apoyos, flexiones de codo, tijeras laterales, } \\
\text { burpees, puente con corredor, remo sentado con banda elástica, "patinador de hielo" y } \\
\text { balanceos con brazos alternados) }\end{array}$ & \\
\hline
\end{tabular}


dietética fue diseñada por un profesional nutricionista.

Semanas 1-2: Según el cuestionario recordatorio 24 horas, se obtuvo un promedio de ingesta de $2.200 \mathrm{kcal} /$ día. En una primera instancia, se realizó un $\Delta$ de solo $-400 \mathrm{kcal}$, obteniendo un régimen de $1.800 \mathrm{kcal} /$ día. Se efectuó una distribución de la molécula calórica (DMC) de $10 \%$ proteínas, $60 \%$ carbohidratos y $30 \%$ lípidos, con el objetivo de generar cambios graduales en la alimentación, considerando hábitos, preferencias y tolerancias alimentarias.

Semanas 3-4: Se mantuvieron $1.800 \mathrm{kcal} /$ día para continuar con la adaptación y adherencia. Sin embargo, se modificó la DMC, siendo 15\% proteínas, 65\% Carbohidratos y 20\% lípidos, con cambios en el tipo de alimentos, tamaño de porciones y horarios.

Semana 5: Al calcular la tasa metabólica basal y considerando un factor promedio de actividad física de 1,3 , se obtuvieron resultados de gasto energético total muy distintos entre las participantes de este grupo. Debido a esto, es que a partir de la semana 5, las mujeres se agruparon según su gasto energético total, para que fuera efectivo el cambio para grupo total. Se estableció un grupo A (6 mujeres) con un promedio de $1.950 \mathrm{kcal} /$ día y grupo B (7 mujeres) con un promedio de 1.700 $\mathrm{kcal} /$ día. Se aplicó un $\Delta$ de $-550 \mathrm{kcal}$, obteniendo una prescripción diaria de $1.400 \mathrm{kcal} /$ día para el grupo A y $1.150 \mathrm{kcal} /$ día para el grupo B. Se realizó una DMC de $20 \%$ proteínas, $60 \%$ carbohidratos y $20 \%$ lípidos.

Semanas 6-9: Se mantuvieron $1.400 \mathrm{kcal} /$ día para el grupo A y $1.150 \mathrm{kcal} /$ día para el grupo B, pero se modificó la DMC, obteniendo $27 \%$ proteínas, 53\% carbohidratos y 20\% lípidos.

Semanas 10-12: Se mantuvieron $1.400 \mathrm{kcal} /$ día para el grupo A y $1.150 \mathrm{kcal} /$ día para el grupo B. En el grupo A, se estableció 32\% proteínas, 48\% carbohidratos y $20 \%$ lípidos, mientras que para el grupo B, se determinó 30\% proteínas, 50\% carbohidratos y $20 \%$ lípidos.

\section{Análisis estadístico}

Para el análisis de los resultados, se hizo una estadística descriptiva, obteniendo medias y desviaciones estándar. Se determinó la normalidad de las variables mediante la prueba de Shapiro-Wilk. Al realizar la prueba en cada una de las variables analizadas, no se rechazó en ninguna de ellas la hipótesis nula de normalidad ( $\mathrm{p}>0,05$ ), por lo que se utilizaron pruebas paramétricas. Para comparar los resultados pre y postest intragrupos, se realizó la prueba T-Student, mientras que para la comparación intergrupos, se utilizó un análisis ANOVA y Chi-Square test, procediendo posteriormente a un análisis Scheffé para determinar entre qué grupos se establecen las diferencias significativas. El análisis estadístico se realizó con el software estadístico STATA/IC 11.2. El valor $\mathrm{p}$ significativo fue $<0,05$.

\section{Resultados}

La Tabla 2 muestra las estadísticas descriptivas de las mujeres que conformaron la muestra $(\mathrm{n}=34)$. No se observan diferencias estadísticas entre los grupos.

En la Figura 1 se presentan los resultados obtenidos en la composición corporal, dinamo-

Tabla 2. Variables descriptivas de los grupos participantes

\begin{tabular}{|lcccc|}
\hline Variables & GC \pm DE & GE \pm DE & GPD \pm DE & p valor \\
\hline Edad (años) & $43,5 \pm 13,9$ & $37,9 \pm 10,3$ & $44,4 \pm 11,4$ & 0,362 \\
\hline Estatura $(\mathrm{cm})$ & $157,5 \pm 5,0$ & $157,3 \pm 6,3$ & $160,2 \pm 4,3$ & 0,274 \\
\hline Peso $(\mathrm{kg})$ & $71,2 \pm 6,8$ & $70,2 \pm 10,8$ & $77,3 \pm 9,7$ & 0,429 \\
IMC $\left(\mathrm{kg} / \mathrm{m}^{2}\right)$ & $28,7 \pm 2,8$ & $28,4 \pm 4,8$ & $30,1 \pm 3,8$ & 0,384 \\
Perímetro cintura $(\mathrm{cm})$ & $93,2 \pm 7,4$ & $93,7 \pm 13,1$ & $100,3 \pm 1,3$ & 0,307 \\
Masa grasa $(\mathrm{kg})$ & $37,9 \pm 4,6$ & $36,5 \pm 5,5$ & $40,4 \pm 4,3$ & 0,466 \\
\hline Masa muscular $(\mathrm{kg})$ & $24,4 \pm 2,6$ & $24,5 \pm 2,7$ & $25,4 \pm 2,7$ & 0,339 \\
\hline
\end{tabular}

GC: grupo control; GE: grupo ejercicio; GPD: grupo prescripción dietética; IMC: índice de masa corporal; : media; D.E.: desviación estándar. Significancia estadística con $p<0,05$ en Chi-Square test. 

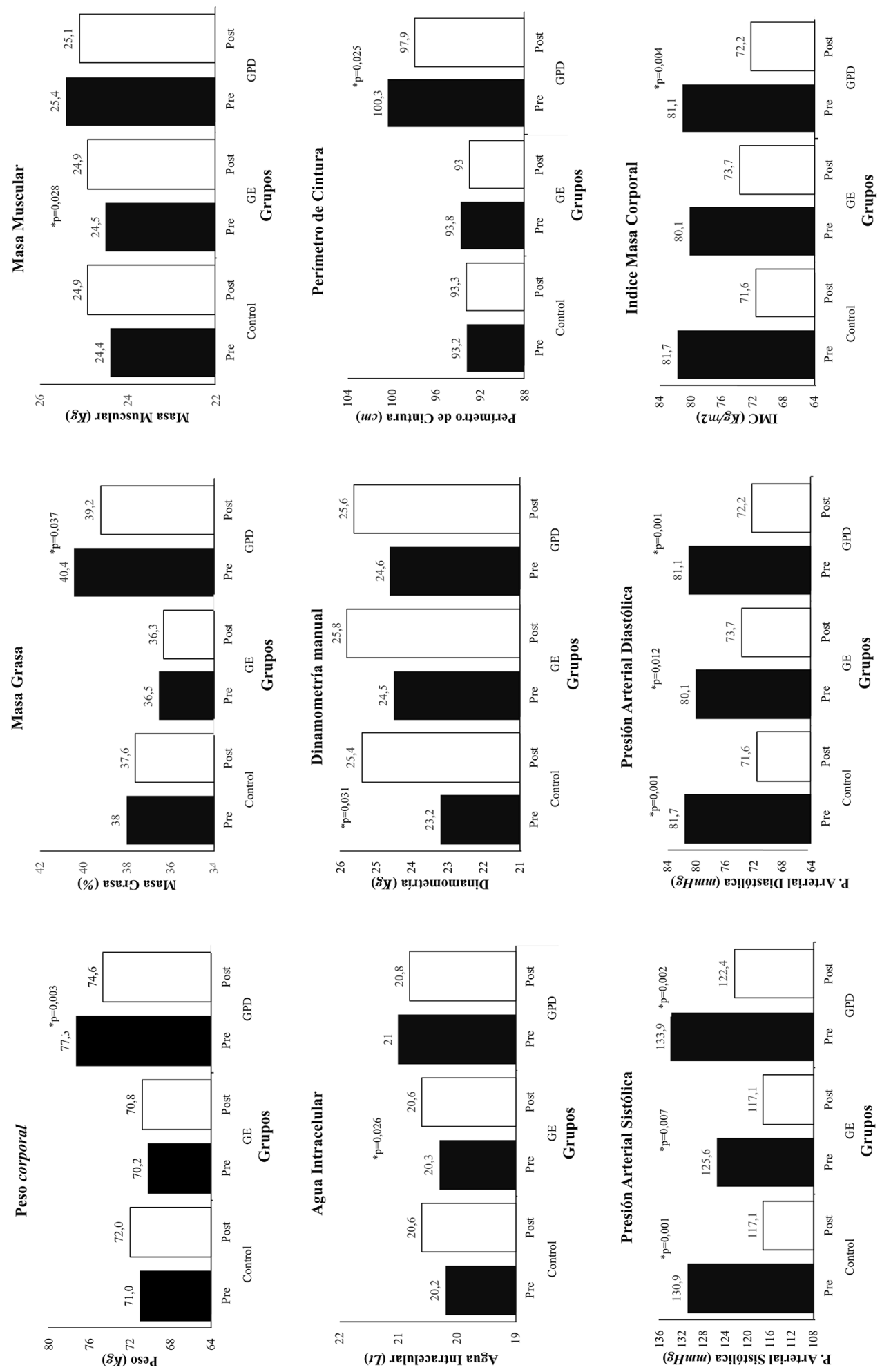
Ejercicio o prescripción dietética sobre la salud en mujeres - F. Rodríguez-Briceño et al

Tabla 3. Diferencias entre los grupos según nivel de cambio (pre-post) en composición corporal, ángulo de fase, dinamometría manual y variables de riesgo cardiovascular

\begin{tabular}{|c|c|c|c|c|c|c|}
\hline \multirow[t]{2}{*}{ Variables } & \multicolumn{2}{|c|}{ GC/GE } & \multicolumn{2}{|c|}{ GC/GPD } & \multicolumn{2}{|c|}{ GE/GPD } \\
\hline & $\Delta$ pre-post & p valor & $\Delta$ pre-post & p valor & $\Delta$ pre-post & p valor \\
\hline \multicolumn{7}{|c|}{ Composición corporal } \\
\hline Peso (Kg) & $+1,0 /+0,6$ & 0,975 & $+1,0 /-2,7$ & $<0,05$ & $+0,6 /-2,7$ & $<0,05$ \\
\hline MG (\%) & $-0,4 /-0,2$ & 0,989 & $-0,4 /-1,2$ & 0,646 & $-0,2 /-1,2$ & 0,537 \\
\hline $\mathrm{MM}(\mathrm{Kg})$ & $+0,5 /+0,4$ & 0,962 & $+0,5 /-0,3$ & 0,127 & $+0,4 /-0,3$ & 0,194 \\
\hline $\operatorname{AIC}(\mathrm{Lt})$ & $+0,4 /+0,3$ & 0,963 & $+0,4 /-0,2$ & 0,135 & $+0,3 /-0,2$ & 0,204 \\
\hline $\mathrm{AEC}(\mathrm{Lt})$ & $+0,2 /+0,1$ & 0,543 & $+0,2 /-0,3$ & $<0,05$ & $+0,1 /-0,3$ & 0,270 \\
\hline $\mathrm{ACT}(\mathrm{Lt})$ & $+0,7 /+0,4$ & 0,948 & $+0,7 /-1,2$ & 0,106 & $+0,4 /-1,2$ & 0,179 \\
\hline $\operatorname{AnF}\left({ }^{\circ}\right)$ & $+0,1 /+0,2$ & 0,995 & $+0,1 / 0,0$ & 0,881 & $+0,2 / 0,0$ & 0,825 \\
\hline \multicolumn{7}{|l|}{ Riesgo CV } \\
\hline $\mathrm{PC}(\mathrm{cm})$ & $+0,1 /-0,8$ & 0,824 & $+0,1 /-2,4$ & 0,218 & $-0,8 /-2,4$ & 0,511 \\
\hline PAS $(\mathrm{mmHg})$ & $-13,8 /-8,5$ & 0,573 & $-13,8 /-11,5$ & 0,934 & $-8,5 /-11,5$ & 0,754 \\
\hline PAD (mmHg) & $-10,1 /-6,4$ & 0,509 & $-10,1 /-8,9$ & 0,876 & $-6,4 /-8,9$ & 0,772 \\
\hline IMC $\left(\mathrm{kg} / \mathrm{m}^{2}\right)$ & $+0,4 /+0,2$ & 0,968 & $+0,4 /-1,0$ & $<0,05$ & $+0,2 /-1,0$ & $<0,05$ \\
\hline \multicolumn{7}{|l|}{ Dinamometría } \\
\hline Absoluta (kg) & $+2,2 /+1,3$ & 0,920 & $+2,2 /+1,0$ & 0,803 & $+1.3 /+1,0$ & 0,970 \\
\hline
\end{tabular}

GC: grupo control; GE: grupo ejercicio; GPD: grupo prescripción dietética; MG: masa grasa; MM: masa muscular; AIC: agua intracelular; AEC: agua extracelular; ACT: agua corporal total; AnF: ángulo de fase; CV: cardiovascular; PC: perímetro de cintura; PAS: presión arterial sistólica; PAD: presión arterial diastólica; IMC: índice de masa corporal. Dinamometría Absoluta: fuerza en $\mathrm{kg}$. Valor significativo $\mathrm{p}<0,05$.

metría manual y variables de riesgo cardiovascular ( $\mathrm{RCV})$, pre y postintervención intragrupos, con sus respectivos valores de significancia estadística.

En cuanto a las variables de composición corporal, en GPD hubo una disminución significativa en peso $(\mathrm{p}=0,003)$ y $\% \mathrm{MG}(\mathrm{p}=0,037)$, mientras que GE tuvo un aumento significativo en $\mathrm{MM}$ $(\mathrm{p}=0,028)$ y $\operatorname{AIC}(\mathrm{p}=0,026)$. En relación con la fuerza por dinamometría, solo el GC presentó un aumento significativo $(\mathrm{p}=0,031)$.

Respecto a las variables de RCV, el GPD tuvo una disminución significativa en todas las medidas analizadas de RCV pre y postintervención: perímetro de cintura $(\mathrm{p}=0,025)$, presión arterial sistólica (PAS) $(\mathrm{p}=0,002)$, presión arterial diastólica (PAD) $(\mathrm{p}=0,001)$ e IMC $(\mathrm{p}=0,004)$. El GE obtuvo una disminución significativa en PAS $(\mathrm{p}=0,007)$ y PAD $(\mathrm{p}=0,012)$. Lo mismo ocurrió con el GC en PAS ( $\mathrm{p}=0,001)$ y PAD $(\mathrm{p}=0,001)$. Al comparar pre y postintervención, el GC presentó diferencias significativas en 3 variables, GE en 4 variables y GPD en 6 variables.

En la Tabla 3, se muestran las comparaciones entre grupos respecto a los resultados obtenidos en la composición corporal, AnF, fuerza muscular y variables de RCV.

En el peso, hubo diferencia significativa entre GPD y GC $(\mathrm{p}=0,004)$ y entre GPD y GE $(\mathrm{p}=0,006)$. El AEC presentó diferencia significativa entre GC y GPD $(\mathrm{p}=0,032)$. Respecto al IMC, hubo diferencia estadística entre GPD y GC $(\mathrm{p}=0,004)$ y entre GPD y GE $(\mathrm{p}=0,007)$.

\section{Discusión}

\section{Composición corporal}

Esta investigación permite observar que una prescripción dietética induce mayor reducción de peso corporal respecto del GE. El peso corporal del GPD tiene una reducción moderada en comparación con otros estudios que también analizan el efecto con dieta hipocalórica ${ }^{12-16}$. Esto podría asociarse al tiempo mayor de intervención (6 meses), en comparación con este estudio (3 meses).

Por otra parte, una dieta hipocalórica puede conllevar una disminución no deseada de MM, 
conduciendo a una reducción del gasto energético. Esta pérdida causaría un efecto compensatorio del gasto energético capaz de obstaculizar la progresión en la pérdida de peso ${ }^{17,18}$. Por tanto, el tratamiento más efectivo no solo será aquel que provoque mayor pérdida de peso y $\mathrm{MG}$, sino también el que permita mantener o aumentar la $\mathrm{MM}^{8}$. Si bien el GPD presenta una disminución en $\mathrm{MM}(-0,3 \mathrm{~kg})$, esta no fue significativa.

Respecto al peso corporal del GE, este aumentó en $0,6 \mathrm{~kg}$, mientras que otros estudios demuestran una reducción de entre $-1,4$ y $-2,2 \mathrm{~kg}^{5,19,20}$. En este caso hubo un incremento significativo en MM $(\mathrm{p}<0,05)$. Este aumento en la $\mathrm{MM}$ en las 12 semanas explica que este grupo no perdiera peso significativamente. No obstante, el aumento de la MM o de masa libre de grasa, se asocia a un aumento en el gasto energético total, gasto energético de reposo y termogénesis independiente a los cambios en el peso corporal neto ${ }^{21}$.

En nuestro estudio, como no fue evaluado el gasto energético por calorimetría, no es posible precisar los cambios que pudo producir el GE.

Respecto a la MG del GE, tuvo una disminución de $-0,2 \%$, un cambio menor respecto otro estudio que aplicó un entrenamiento en circuito de tres veces/semana por 3 meses, a una intensidad de 70-85\% de la FCmax, donde disminuyó el \%MG en $-4,5 \%{ }^{22}$. Esta reducción significativa pudo ser provocada por la mayor intensidad aplicada en la intervención.

El GE también presentó un aumento significativo en AIC $(p<0,05)$, por lo que, posiblemente, hubo cambios en la masa celular corporal. Además, si bien el aumento del ACT no fue significativo, se ha estudiado que el ACT aumenta al incrementar la $\mathrm{MM}^{23}$.

Respecto al AnF, no hubo cambios en ninguno de los grupos. No obstante, los valores de AnF obtenidos por todos los grupos, se encuentran entre las referencias de normalidad $\left(5^{\circ}-7^{o}\right)^{24-25}$.

$\mathrm{Si}$ bien el aumento en AnF no es significativo en GE, este incremento podría explicarse según lo propuesto por Adelman ${ }^{26}$, quien concluye que esta variable aumenta a medida que se incrementa el IMC, debido a un aumento de la masa celular $\mathrm{y} \mathrm{MM}^{27}$.

\section{Fuerza muscular}

Se ha establecido previamente una asociación positiva entre AnF y fuerza de presión manual, siendo cualquiera de los dos un importante marcador de la calidad muscular y del estado de salud ${ }^{28-30}$.

En este estudio, solo el GC obtuvo un incremento significativo $(+2,2 \mathrm{~kg} ; \mathrm{p}<0,05)$.

Un reciente estudio demostró cambios positivos en la fuerza por dinamometría manual en una intervención de 15 semanas de duración, pero con un alto estímulo de ejercicio (90 min por 6 veces/ semana $)^{31}$. Ramírez et al. ${ }^{32}$ obtuvieron resultados similares en un grupo de mujeres adultas, en un programa de actividad física de 5 meses, 3 días/ semana alternos y $60 \mathrm{~min} /$ sesión a intensidad entre $60-70 \%$ de la FcR.

En este estudio, el aumento de la fuerza de presión manual absoluta en kg del GE y GPD no fueron significativos. Tampoco se apreciaron diferencias al comparar los cambios del pre y postest entre los grupos (Tabla 3). Al respecto, un metaanálisis que analizó diez estudios de intervención con restricción dietética demostró que en siete de ellos hubo una disminución de la fuerza manual promedio de $-2,4 \mathrm{~kg}(\mathrm{p}=0,046)$, lo que representa $4,6 \%$ respecto de los valores basales, asociando la pérdida de peso con la disminución de la fuerza muscular $^{33}$. En relación al GE, si bien tuvo una mejora promedio de $+1,0 \mathrm{~kg}$, esta no fue significativa, lo que puede atribuirse a un insuficiente tiempo de intervención y a que el tipo de ejercicio desarrollado (en circuito) no fue el adecuado.

\section{Riesgo cardiovascular}

Al comparar los valores de IMC pre y postest, tanto GC como GE muestran un aumento $(0,4$ $\mathrm{kg} / \mathrm{m}^{2}$ y $0,2 \mathrm{~kg} / \mathrm{m}^{2}$, respectivamente), mientras que GPD es el único que presenta una disminución significativa $(\mathrm{p}=0,004)$, equivalente $\mathrm{a}-1 \mathrm{~kg} / \mathrm{m}^{2}$.

El GPD presenta un promedio en IMC pretest de $30,1 \mathrm{~kg} / \mathrm{m}^{2}$, por lo que tendría mayor RCV previo a la intervención en comparación a los otros grupos. No obstante, postintervención, este grupo disminuyó el promedio $\left(29,1 \mathrm{~kg} / \mathrm{m}^{2}\right)$, por lo que reduciría el RCV, ya que mientras mayor sea la reducción de IMC, mayor serán los beneficios a nivel cardiovascular ${ }^{34}$. Esta disminución se asocia principalmente a los cambios realizados en los hábitos alimentarios.

Respecto al perímetro de cintura, tanto GE como GPD presentan una disminución $(-0,8 \mathrm{~cm}$ y $-2,4 \mathrm{~cm}$, respectivamente), sin embargo, es significativa solo en GPD ( $p=0,025)$, lo cual se condice con la pérdida significativa en peso corporal y MG, 
posiblemente, debido al régimen hipocalórico prescrito. Respecto a la PA, los tres grupos presentan una disminución significativa tanto en PAS como PAD postintervención $(\mathrm{p}<0,05)$.

\section{Fortalezas y limitaciones}

Se destaca como principal fortaleza la aplicación de una estrategia de intervención por 12 semanas en grupos cautivos y que incluyó a un grupo control. Como limitaciones se destaca el reducido número de participantes que aceptaron participar de las intervenciones, una muestra no aleatorizada y la no evaluación de la condición cardiorrespiratoria.

\section{Conclusión}

Los datos aportados en este estudio señalan que un programa de entrenamiento en circuito de tipo mixto, durante 12 semanas, produce cambios significativos en aumento de la MM y AIC, y disminuye la presión arterial en mujeres adultas con SP u OB. En cambio, una prescripción dietética durante 12 semanas tiene efectos significativos en la disminución del peso corporal, MG, IMC, perímetro de cintura y presión arterial. Realizar intervenciones que incluyan ejercicio y prescripción dietética podrían maximizar los efectos positivos sobre la salud.

Agradecimientos: A quienes aceptaron ser parte del estudio por su participación, al profesor Héctor Carriel que ejecutó el programa de actividad física en la Escuela de Salud de la Universidad Tecnológica de Chile INACAP, Valparaíso y a estudiantes de Nutrición y Dietética de esta universidad que apoyaron en la recolección de los datos.

\section{Referencias}

1. WHO. Obesity: preventing and managing the global epidemic: Report of a WHO Consultation. Geneva. WHO, 2000. Disponible en: https://www.who.int/nutrition/ publications/obesity/WHO_TRS_894/en/

2. Ministerio de Salud. Chile. Encuesta nacional de salud ENS Chile 2016-2017. Disponible en: https://www. minsal.cl/wp-content/uploads/2017/11/ENS-201617_PRIMEROS-RESULTADOS.pdf.

3. Ministerio del Deporte. Chile. Encuesta Nacional de Actividad Física y Deporte en Población de 18 años y más. Disponible en: http://www.mindep.cl/encuesta-actividad-fisica-y-deporte-2018/

4. Nicklas BJ, Wang X, You T, Lyles MF, Demons J, Easter $L$, et al. Effect of exercise intensity on abdominal fat loss during calorie restriction in overweight and obese postmenopausal women: a randomized, controlled trial. Am J Clin Nutr 2009; 89 (4): 1043-52. DOI: 10.3945/ ajcn.2008.26938.

5. Church TS, Martin CK, Thompson AM, Earnest CP, Mikus CR, Blair SN. Changes in weight, waist circumference and compensatory responses with different doses of exercise among sedentary, overweight postmenopausal women. PLoS One 2009; 4 (2): e4515. DOI: 10.1371/ journal.pone.0004515.

6. Thorogood A, Mottillo S, Shimony A, Filion KB, Joseph $\mathrm{L}$, Genest J, et al. Isolated aerobic exercise and weight loss: a systematic review and meta-analysis of randomized controlled trials. Am J Med 2011; 124 (8): 747-55. DOI: 10.1016/j.amjmed.2011.02.037.

7. Burgos C, Henríquez C, Cerda H, Ramírez R. Entrenamiento físico, exceso de peso y obesidad. En: Mahecha S. Actividad física y ejercicio en salud y enfermedad. Santiago, Chile: Mediterráneo; 2017. p. 289-96.

8. Bonfanti N, Fernández JM, Gomez-Delgado F, Pérez-Jiménez F. Effect of two hypocaloric diets and their combination with physical exercise on basal metabolic rate and body composition. Nutr Hosp 2014; 29 (3): 635-43. DOI: 10.3305/nh.2014.29.3.7119.

9. Cabañas MD, Esparza F. Compendio de Cineantropometría. Madrid, España: CTO Editorial. 2009.

10. Kyle UG, Bosaeus I, De Lorenzo AD, Deurenberg P, Elia M, Gómez JM, et al. Bioelectrical impedance analysis-part II: utilization in clinical practice. Clin Nutr 2004; 23 (6): 1430-53. DOI: 10.1016/j.clnu.2004.09.012.

11. Ministerio de Salud. Chile. Guía Clínica Hipertensión arterial primaria o esencial en personas de 15 años y más 2010. Disponible en: https://www.minsal.cl/portal/url/ item/7220fdc4341c44a9e04001011f0113b9.pdf.

12. Wing RR, Venditti E, Jakicic JM, Polley BA, Lang W. Lifestyle intervention in overweight individuals with a family history of diabetes. Diabetes Care 1998; 21 (3): 350-9.

13. Jones DW, Miller ME, Wofford MR, Anderson DC, Cameron ME, Willoughby DL, et al. The effect of weight loss intervention on antihypertensive medication requirements in the hypertension Optimal Treatment (HOT) study. Am J Hypertens 1999; 12 (12): 1175-80.

14. Rapoport L, Clark M, Wardle J. Evaluation of a modified cognitive-behavioural programme for weight management. Int J Obes Relat Metab Disord 2000; 24 (12): 1726-37. 
15. Bacon L, Keim NL, Van Loan MD, Derricote M, Gale B, Kazaks A, et al. Evaluating a 'non-diet'wellness intervention for improvement of metabolic fitness, psychological well-being and eating and activity behaviors. Int J Obes Relat Metab Disord 2002; 26 (6): 854-65. DOI: 10.1038/ sj.ijo.0802012.

16. Wolf AM, Conaway MR, Crowther JQ, Hazen KY, Nadler JL, Oneida B, et al. Translating lifestyle intervention to practice in obese patients with type 2 diabetes: Improving Control with Activity and Nutrition (ICAN) study. Diabetes Care 2004; 27 (7): 1570-6.

17. Stiegler P, Cunliffe A. El papel de la dieta y el ejercicio para el mantenimiento de la masa libre de grasa y la tasa metabólica en reposo durante la pérdida de peso. Medicina deportiva 2006; 36 (3): 239-62.

18. Chaston TB, Dixon JB, O'Brien PE. Changes in fatfree mass during significant weight loss: a systematic review. Int J Obes 2007; 31 (5): 743-50. DOI: 10.1038/ sj.ijo.0803483.

19. Ho SS, Dhaliwal SS, Hills AP, Pal S. The effect of 12 weeks of aerobic, resistance or combination exercise training on cardiovascular risk factors in the overweight and obese in a randomized trial. BMC Public Health 2012; 12 (1): 704. DOI: 10.1186/1471-2458-12-704.

20. Choo J, Lee J, Cho JH, Burke LE, Sekikawa A, Jae SY. Effects of weight management by exercise modes on markers of subclinical atherosclerosis and cardiometabolic profile among women with abdominal obesity: a randomized controlled trial. BMC Cardiovasc Disord. 2014; 14 (1): 82. DOI: 10.1186/1471-2261-14-82.

21. Müller MJ, Enderle J, Bosy-Westphal A. Changes in energy expenditure with weight gain and weight loss in humans. Curr Obes Rep 2016; 5 (4): 413-23.

22. Ahmadizad S, Haghighi AH, Hamedinia MR. Effects of resistance versus endurance training on serum adiponectin and insulin resistance index. Eur J Endocrinol 2007; 157 (5): 625-31. DOI: 10.1530/EJE-07-0223.

23. Martinoli R, Mohamed EI, Maiolo C, Cianci R, Denoth F, Salvadori S, et al. Total body water estimation using bioelectrical impedance: a meta-analysis of the data available in the literature. Acta Diabetol 2003; 40 (1): s203-6. DOI: 10.1007/s00592-003-0066-2.

24. Barbosa-Silva MCG, Barros AJ, Wang J, Heymsfield SB, Pierson Jr RN. Bioelectrical impedance analysis: population reference values for phase angle by age and sex. Am J Clin Nutr 2005; 82 (1): 49-52. DOI: 10.1093/ ajcn.82.1.49.

25. Bosy-Westphal A, Danielzik S, Dörhöfer RP, Later W, Wiese S, Müller MJ. Phase angle from bioelectrical impedance analysis: population reference values by age, sex, and body mass index. J Parenter Enteral Nutr 2006; 30 (4): 309-16. DOI: 10.1177/0148607106030004309.

26. Adelman RD. Obesity and renal disease. Curr Opin Nephrol Hypertens 2002; 11 (3): 331-5.

27. Berral-Aguilar F, Berral de la Rosa F. Impedancia bioeléctrica. En Rodríguez-Rodríguez F, Almagia A. Evaluación y ciencia de la morfoestructura humana. Chile: Ediciones Universitarias de Valparaíso; 2016. p. 177-80.

28. Norman K, Smoliner C, Valentini L, Lochs H, Pirlich $M$. Is bioelectrical impedance vector analysis of value in the elderly with malnutrition and impaired functionality? Nutrition 2007; 23 (7-8): 564-9. DOI: 10.1016/j. nut.2007.05.007.

29. Rodríguez-Rodríguez F, Cristi-Montero C, González-Ruíz K, Correa-Bautista JE, Ramírez-Vélez R. Bioelectrical impedance vector analysis and muscular fitness in healthy men. Nutrients 2016; 8 (7): 407. DOI: 10.3390/nu8070407.

30. Reis BC, de Branco F, Pessoa DF, Barbosa CD, dos Reis AS, de Medeiros LA, et al. Phase Angle Is Positively Associated With Handgrip Strength in Hospitalized Individuals. Topics in Clinical Nutrition 2018; 33 (2): 127-33. DOI: 10.1097/TIN.0000000000000135.

31. Singh K, Bhatti GK. (2020). Effect of physical exercise training to improve physical fitness in overweight middle-aged women. International Journal of Scientific Research, 9 (1).

32. Ramírez A, Olcina GJ, Muñoz D, Timón R, Robles M, Maynar M. Efectos de un programa de actividad física sobre la condición física en mujeres premenopáusicas. Actividad física y salud 2012; $n^{\circ}$ 109: 25-31.

33. Zibellini J, Seimon RV, Lee CMY, Gibson AA, Hsu $\mathrm{MSH}$, Sainsbury A. Effect of diet-induced weight loss on muscle strength in adults with overweight or obesity-a systematic review and meta-analysis of clinical trials. Obesity Reviews 2016; 17 (8): 647-63.

34. Labraña AM, Durán E, Martínez MA, Leiva AM, Garrido-Méndez A, Díaz X, et al. Menor peso corporal, de índice de masa corporal y de perímetro de cintura se asocian a una disminución en factores de riesgo cardiovascular en población chilena. Rev Med Chile 2017; 145 (5): 585-94.

35. Tanaka H, Monahan KD, Seals DR. Age-predicted Maximal Heart Rate Revisited. J Am Coll Cardiol 2001; 37 (1): 153-6.

36. Karvonen J, Vuorimaa T. Heart Rate and Exercise Intensity During Sports Activities. Practical Application. Sports Med 1988; 5 (5): 303-11. 